\section{Transgene silencing}

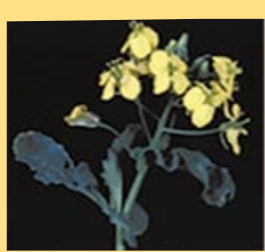

The 35 s promoter from the cauliflower mosaic virus (CaMV) is used frequently to control transgene expression in plants. Now on page 995, Al-Kaff et al. have shown that a $35 \mathrm{~S}$ promoter-driven transgene encoding herbicide resistance in oilseed rape plants can be downregulated following infection of the plants with CaMV. The silencing of the transgene and herbicide tolerance could be triggered by different CaMV pathogenic variants and at different stages of plant development. Because CaMV is a prevalent pathogen worldwide, these results have implications for the stability of $35 \mathrm{~S}$ promoter-driven transgene expression in transgenic crops. MS

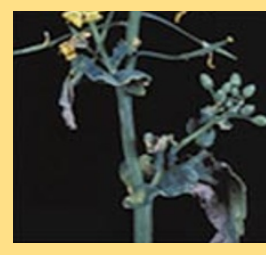

\section{Spinal cord gene therapy}

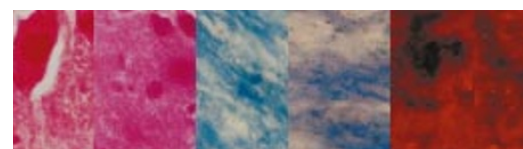

On page 964, Bledsoe et al. demonstrate that by taking advantage of the strong natural tropism of poliovirus for motor neurons of the CNS, they could deliver therapeutic proteins to the spinal cord of mice expressing the human poliovirus receptor. They engineered poliovirus replicons to express foreign genes in place of capsid proteins, which rendered them noninfectious and only capable of replicating when capsid proteins were provided in trans by vaccinia virus. The researchers injected mice intraspinally with the poliovirus vectors encoding either GFP or the cytokine TNF$\alpha$. As expected, TNF- $\alpha$ produced diverse symptoms as a result of its biological activity, whereas GFP seemed innocuous, suggesting that the poliovirus replicon itself does not harm the targeted tissue. Targeted gene therapy for the CNS could potentially be used to treat spinal cord trauma and neurological disease.

\section{Improving antigen delivery}

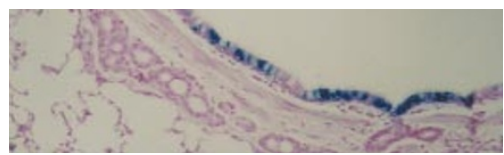

Professional antigen-presenting cells (APCs) are potent stimulators of T-cell-mediated immune responses. On page 974, Chattergoon et al. describe a method for enhancing cytotoxic T-lymphocyte responses to an antigen of choice. They co-injected DNA encoding the desired antigen along with DNA encoding the Fas protein (a receptor that plays a role in cell death mechanisms) into the muscle of mice. The Fasmediated death of these antigen-bearing cells resulted in increased antigen acquisition by APCs and enhanced antigen-specific cytotoxic T-lymphocyte responses. Increasing delivery of antigen to APCs could potentially improve the potency of immunotherapies. $M S$

\section{Technical Reports}

On page 1001, Lindblad-Toh et al. demonstrate that single-nucleotide polymorphism (SNP) array hybridization is an accurate and efficient approach for evaluating genome-wide tumor loss of heterozygosity (LOH). The strategy uses 24-multiplex PCR reactions to amplify polymorphic regions from tumor samples, followed by hybridization to a human SNP chip to determine the genotypes for nearly 1,500 genome-wide markers. The results are then compared to genotypes obtained from nontumor samples from the same individuals. The authors demonstrate that the microarray-based approach offers advantages in throughput relative to other methodologies, by decreasing the amount of sample required and an increasing the number of loci studied.

Staphylococcal $\alpha$-hemolysin proteins form membrane pores that can be engineered to detect the concentration and identity of analytes, even at the singlemolecule level. Bayley and colleagues now describe a method to use engineered $\alpha$-hemolysin with ion-binding sites built into its barrel for simultaneously sensing the concentrations of two or more divalent metal ions in solution. (See p. 1005).

ND 